\section{Isolated Tumor Cells in Lymph Nodes of Patients with Gastric Cancer}

\section{TO THE EDITORS:}

We appreciate Dr. Roukos' interest in our article on isolated tumor cells (ITC) in lymph nodes of gastric cancer patients and would like to respond to his comments.

In patients with recurrent disease, there must have been residual tumor cells in the form of occult micrometastases left behind at the time of apparent curative surgery. In recent years we have focused on studies of ITC in gastric cancer. In the article published in the Annals of Surgical Oncology, the presence of ITC in lymph nodes was not shown to be an adverse prognostic factor and did not provide any valuable information for therapeutic strategies. ${ }^{1}$ The mere presence of ITC in the bone marrow and peripheral blood of gastric cancer patients did not correlate with clinical stage in our large-scale study. ${ }^{2}$ However, expression of vascular endothelial growth factor receptor1(VEGFR-1), urokinase-type plasminogen activator receptor (UPAR), membrane type 1 matrix metalloproteinase (MT1-MMP), and ID1 were shown to be predictive factors for distant metastases in our case series. ${ }^{2-5}$ These studies, which can make some contribution to oncological science, are based on conventional comparative effectiveness research (CER), although they cannot eventually overcome the heterogeneity and diversity in cancer therapy.

In general, we basically agree with the proposals of Dr. Roukos. Current advances in quantitative genetics, wholegenome scans, personal genomics, and system biology provide exciting perspectives towards genotype-phenotype relationship prediction. The accuracy and reproducibility of microarray-based clinical research have been discussed, and guidelines for statistical analysis and reporting for clinical microarray studies have recently been validated. ${ }^{6}$ The introduction of novel personalized cancer diagnostics and therapeutics in the near future is a realistic goal.
When we manage clinical issues, no matter what technological advances may occur, the analysis will be limited by the accuracy of clinical data and availability of collected samples. ${ }^{7}$ If the study is well designed in these terms, whether the methodology is a comprehensive surveillance or candidate-gene approach, valuable results will finally be achieved.

Takeo Fukagawa, MD, Hitoshi Katai, MD, and Mitsuru Sasako, MD

Gastric Surgery Division, National Cancer Center Hospital, Tokyo, Japan

e-mail: tfukagaw@ncc.go.jp

Published Online: 10 October 2009

(C) Society of Surgical Oncology 2009

\section{REFERENCES}

1. Fukagawa T, Sasako M, Shimoda T, et al. The prognostic impact of isolated tumor cells in lymph nodes of T2N0 gastric cancer: comparison of American and Japanese gastric cancer patients. Ann Surg Oncol. 2009;16(3):609-13.

2. Mimori K, Fukagawa T, Kosaka Y, et al. Hematogenous metastasis in gastric cancer requires isolated tumor cells and expression of vascular endothelial growth factor receptor-1. Clin Cancer Res. 2008;14(9):2609-16.

3. Kita Y, Fukagawa T, Mimori K, et al. Expression of uPAR mRNA in peripheral blood is a favourite marker for metastasis in gastric cancer cases. Br J Cancer. 2009;100(1):153-9.

4. Mimori K, Fukagawa T, Kosaka Y, et al. A large-scale study of MT1-MMP as a marker for isolated tumor cells in peripheral blood and bone marrow in gastric cancer cases. Ann Surg Oncol. 2008;15(10):2934-42.

5. Iwatsuki M, Fukagawa T, Mimori K, et al. Bone marrow and peripheral blood expression of ID1 in human gastric carcinoma patients is a bona fide indicator of lymph node and peritoneal metastasis. Br J Cancer. 2009;100(12):1937-42.

6. Dupuy A, Simon RM. Critical review of published microarray studies for cancer outcome and guidelines on statistical analysis and reporting. J Natl Cancer Inst. 2007;99(2):147-57.

7. Roses AD. Pharmacogenetics in drug discovery and development: a translational perspective. Nat Rev Drug Discov. 2008;7(10):807-17. 